This is the pre-peer reviewed version of the following article: Rihel J and Schier AF (2012). Behavioral screening for neuroactive drugs in zebrafish. Developmental Neurobiology, 72(3):373-385., which has been published in final form at http://onlinelibrary.wiley.com/doi/10.1002/dneu.20910/full

\title{
Behavioral Screening for Neuroactive Drugs in Zebrafish
}

Jason Rihel ${ }^{1}$ and Alexander F. Schier ${ }^{1,2,3,4}$

${ }^{1}$ Department of Molecular and Cellular Biology, ${ }^{2}$ Division of Sleep Medicine, ${ }^{3}$ Center for Brain Science, ${ }^{4}$ Harvard Stem Cell Institute; Harvard University, Cambridge, MA 02138, USA

e-mail: schier@fas.harvard.edu, rihel@fas.harvard.edu

Tel: 617.496.4835 Fax: 617.495 .9300

\section{Summary}

The larval zebrafish has emerged as a vertebrate model system amenable to small molecule screens for probing diverse biological pathways. Two large-scale small molecule screens examined the effects of thousands of drugs on larval zebrafish sleep/wake and photomotor response behaviors. Both screens identified hundreds of molecules that altered zebrafish behavior in distinct ways. The behavioral profiles induced by these small molecules enabled the clustering of compounds according to shared phenotypes. This approach identified regulators of sleep/wake behavior and revealed the biological targets for poorly characterized compounds. Behavioral screening for neuroactive small molecules in zebrafish is an attractive complement to in vitro screening efforts, because the complex interactions in the vertebrate brain can only be revealed in vivo.

\section{The problem of psychotropic drug discovery}

It is estimated that more than 60 million Americans and 450 million people worldwide suffer from some form of mental illness, but the search for new drugs to alleviate psychiatric and central 
This is the pre-peer reviewed version of the following article: Rihel J and Schier AF (2012). Behavioral screening for neuroactive drugs in zebrafish. Developmental Neurobiology, 72(3):373-385., which has been published in final form at http://onlinelibrary.wiley.com/doi/10.1002/dneu.20910/full

nervous system (CNS) disorders is in crisis (Agid et al., 2007; Miller, 2010). Compared to drugs in other therapy categories, the development of CNS drugs costs more (US \$849 million per compound), and their approval takes longer (an average of 8.8 years) and is more difficult (approval rate of 8.2\%) (Pangalos et al., 2007; Miller, 2010). Even more problematic is the dearth of innovative ideas to discover new drugs. Many compounds currently in clinical trials merely repurpose approved structures for slightly different therapeutic indications. Other leads provide slightly greater efficacy on well-trodden single disease targets with questionable therapeutic relevance. The lack of progress is not surprising, considering that tools for highthroughput in vivo drug discovery and characterization are not available. In vitro screening assays have identified compounds with improved binding efficacy on specific targets, but in vitro studies do not reliably predict therapeutic outcomes in vivo (Geddes et al., 2000; FischerBarnicol et al., 2008). Indeed, most effective psychoactive compounds were serendipitously discovered decades ago in whole-animal behavioral contexts (Wong et al., 2005; Ban, 2006; Kokel and Peterson, 2008).

An alternative to in vitro target-based screens is phenotype-based, whole organism screening. Whole organism screens keep intact the complex architecture of the brain's signaling networks. Equally important, whole-organism screens do not require well-validated targets to discover compounds that give desirable phenotypic outcomes. Although the benefit of systematic in vivo screens is well recognized, it is cost- and time- prohibitive to implement such screens in mammals. For example, the Squibb anti-tuberculosis screen that discovered isoniazid required the phenotyping of more than 5000 compounds in mice (reviewed in (Zon and Peterson, 2005; Kokel and Peterson, 2008)). Recent studies in zebrafish suggest that this model organism can be used for the high-throughput behavioral screening for neuroactive 
This is the pre-peer reviewed version of the following article: Rihel J and Schier AF (2012). Behavioral screening for neuroactive drugs in zebrafish. Developmental Neurobiology, 72(3):373-385., which has been published in final form at http://onlinelibrary.wiley.com/doi/10.1002/dneu.20910/full

molecules and complement in vitro and mammalian systems.

\section{Small Molecule Screening in Zebrafish}

Long recognized for their powerful genetics, the zebrafish has emerged in recent years as a cost-effective model system for whole organism small molecule screening. Several features of zebrafish biology that enable genetic studies are also valuable for small molecule screens. Zebrafish are cheap to grow in the laboratory. A single breeding pair can produce hundreds of fertilized embryos per week. These embryos develop rapidly into a free-swimming larva with complex behaviors as early as 4 days post-fertilization. The embryos and larvae are small and can easily be transferred into 96-well plates for high-throughput developmental and behavioral assays. Each step of a zebrafish screen can now be fully automated, from loading, orienting, imaging, and laser-dissection (Pardo-Martin et al., 2010). In addition, small molecules can be added directly to the water and readily taken up by the fish through the gills or skin. This straightforward delivery of bioactive compounds not only facilitates high-throughput automation of assays but also provides exquisite control over experimental timing to avoid developmental effects.

Since Peterson and colleague's proof of principle study on developmental perturbagens (Peterson et al., 2000), many small molecule screens have been conducted in zebrafish, especially in the context of developmental processes. Because these screens have recently been thoroughly reviewed elsewhere (MacRae and Peterson, 2003; Peterson et al., 2004; Zon and Peterson, 2005; Wheeler and Brandli, 2009; Peal et al., 2010; Strahle and Grabher, 2010; Taylor et al., 2010), we will only highlight a few important examples here. In one striking early success, a small molecule screen identified two chemical suppressors of the zebrafish gridlock mutation (in the transcriptional repressor hey2 gene), which disrupts normal aorta development 
This is the pre-peer reviewed version of the following article: Rihel J and Schier AF (2012). Behavioral screening for neuroactive drugs in zebrafish. Developmental Neurobiology, 72(3):373-385., which has been published in final form at http://onlinelibrary.wiley.com/doi/10.1002/dneu.20910/full

and leads to a lack of circulation to the trunk and tail (Peterson et al., 2004). The suppressors did not directly affect the gridlock disease gene but instead acted to upregulate vascular endothelial growth factor (VEGF), which promotes blood vessel formation. Thus, not only can whole organism screens identify small molecules that can rescue a genetic disease, but they can also highlight novel disease-relevant molecular pathways that in vitro assays would likely miss.

In another study, a zebrafish small molecule screen identified the prostaglandin pathway as a regulator of hematopoietic stem cell (HSC) number in vivo, an effect that is conserved in mice and may have clinical applications to enhance HSC number in cord blood transplants (Lord et al., 2007; North et al., 2007). This screen also identified nitric oxide signaling as an important regulator of HSC number in zebrafish and revealed the importance of blood flow in promoting HSC numbers (North et al., 2009). These experiments demonstrate another power of small molecule screens - the parallel nature of chemical screens allows for the simultaneous manipulation and study of numerous biological pathways.

Other developmental events that have been investigated by small molecule screens in zebrafish include cell cycle regulation (Murphey et al., 2006), FGF signaling (Molina et al., 2009), BMP signaling (Yu et al., 2008), hair cell death (Owens et al., 2008; Ou et al., 2009), retinal blood vessel growth (Kitambi et al., 2009), melanocyte biology (Budi et al., 2008; Hultman et al., 2008), cancer biology (Yeh et al., 2009), heart rhythms (Peal et al., 2011), dietary lipid absorption (Clifton et al., 2010) and tissue and fin regeneration (Mathew et al., 2007; Oppedal and Goldsmith, 2010). Clearly, many biological processes in zebrafish are amenable to high throughput small molecule screens. 
This is the pre-peer reviewed version of the following article: Rihel J and Schier AF (2012). Behavioral screening for neuroactive drugs in zebrafish. Developmental Neurobiology, 72(3):373-385., which has been published in final form at http://onlinelibrary.wiley.com/doi/10.1002/dneu.20910/full

\section{Probing Zebrafish Behavior with Small Molecules}

Recent experiments in both larval and adult zebrafish have tested the behavioral effects of psychotropic compounds, including drugs of abuse (Gerlai et al., 2000; Darland and Dowling, 2001; Bilotta et al., 2002; Dlugos and Rabin, 2003; Lockwood et al., 2004; Gerlai et al., 2006;

Lau et al., 2006; Gerlai et al., 2008; Kily et al., 2008; Lopez-Patino et al., 2008; Lopez Patino et al., 2008; Egan et al., 2009; Fernandes and Gerlai, 2009; Gerlai et al., 2009; MacPhail et al., 2009; Webb et al., 2009; Blaser et al., 2010; Cachat et al., 2010; Irons et al., 2010; Sackerman et al., 2010; Wong et al., 2010; Dlugos et al., 2011; Mathur and Guo, 2011; Maximino et al., 2011), anxiolytics and anxiogenics (Levin et al., 2007; Bencan and Levin, 2008; Bencan et al., 2009; Egan et al., 2009; Lau et al., 2011; Maximino et al., 2011), anti-psychotics (Giacomini et al., 2006; Boehmler et al., 2007), hallucinogens (Swain et al., 2004; Blank et al., 2009; Grossman et al., 2010; Seibt et al., 2010), and sedatives (Zhdanova et al., 2001; Ruuskanen et al., 2005; Renier et al., 2007). Conditioned placed preference testing in adults has been used to identify zebrafish mutants with defects in cocaine (Darland and Dowling, 2001) or amphetamine (Webb et al., 2009) reward pathways. The effects of both acute and chronic exposure of ethanol have been extensively studied in adult locomotion (Gerlai et al., 2000; Gerlai et al., 2008), reward (Kily et al., 2008), tolerance (Dlugos and Rabin, 2003; Blaser et al., 2010; Dlugos et al., 2011), withdrawal (Gerlai et al., 2009; Cachat et al., 2010), aggression (Gerlai et al., 2000), and anxiety tests (Gerlai et al., 2006; Fernandes and Gerlai, 2009; Sackerman et al., 2010; Wong et al., 2010; Mathur and Guo, 2011; Maximino et al., 2011). Anxiolytics, including nicotine (Levin et al., 2007; Bencan and Levin, 2008), buspirone, diazepam, and fluoxetine (Bencan et al., 2009; Maximino et al., 2011), and anxiogenics such as caffeine (Egan et al., 2009) alter adult zebrafish anxiety-related behavioral responses as assessed by either a novel tank or light/dark preference paradigms. Drugs that affect adult zebrafish learning and memory tests include nicotine (Levin and Chen, 2004; Levin et al., 2006), the anti-cholinergic 
This is the pre-peer reviewed version of the following article: Rihel J and Schier AF (2012). Behavioral screening for neuroactive drugs in zebrafish. Developmental Neurobiology, 72(3):373-385., which has been published in final form at http://onlinelibrary.wiley.com/doi/10.1002/dneu.20910/full

scopolamine (Kim et al., 2010; Richetti et al., 2011), the anti-histaminerigc $\alpha$ fluoromethylhistidine (Peitsaro et al., 2003), the NMDA receptor antagonist MK-801 (Swain et al., 2004; Blank et al., 2009), environmental neurotoxins (Smith et al., 2010), and melatonin (Rawashdeh et al., 2007). Some work has also been done on the effects of neuroactive drugs on larval zebrafish locomotor behavior. Ethanol and amphetamine increase locomotor activity at low doses but inhibit locomotion at high doses (Lockwood et al., 2004; MacPhail et al., 2009; Irons et al., 2010). The anti-psychotics fluphenazine, haloperidol, and clozapine cause larval movement defects (Giacomini et al., 2006; Boehmler et al., 2007). Finally, several sedatives, including melatonin, benzodiazepines, barbiturates, histamine receptor $\mathrm{H} 1$ antagonists, and $\alpha-2$ adrenergic agonists also reduce larval locomotion (Zhdanova et al., 2001; Ruuskanen et al., 2005; Renier et al., 2007). These studies collectively demonstrate the power of probing complex zebrafish behaviors with neuroactive small molecules.

\section{Behavioral Small Molecule Screens}

The first two large-scale screens for small molecules that modulate behavior have demonstrated the potential of zebrafish for psychotropic drug discovery (Kokel et al., 2010; Rihel et al., 2010a). Rihel et al. (2010) screened the effects of nearly 4000 small molecules on zebrafish sleep/wake behaviors, while Kokel et al. (2010) tested how 14000 drugs altered larval responses to a high intensity light pulse. Both studies leveraged the 96 -well plate format to study behavior at high throughput and introduced the concept of clustering active compounds by their behavioral profiles. The quantitative, multi-dimensional behavioral profiles of each small molecule were used to identify unexpected relationships among molecules with similar behavioral outputs (Figure 1). 
This is the pre-peer reviewed version of the following article: Rihel J and Schier AF (2012). Behavioral screening for neuroactive drugs in zebrafish. Developmental Neurobiology, 72(3):373-385., which has been published in final form at http://onlinelibrary.wiley.com/doi/10.1002/dneu.20910/full

\section{Sleep/Wake Screen}

Zebrafish larvae and adults have behavioral, pharmacological, anatomical, molecular, and genetic correlates of mammalian sleep (Zhdanova et al., 2001; Kaslin et al., 2004; Faraco et al., 2006; Prober et al., 2006; Zhdanova, 2006; Yokogawa et al., 2007; Appelbaum et al., 2009; Rihel et al., 2010b). First, zebrafish exhibit locomotor patterns characteristics of sleep-like states. Larvae rest predominantly at night, under endogenous circadian control (Hirayama et al., 2005). During these rest bouts, they have increased arousal thresholds, an important criterion for behaviorally defined sleep-like states (Zhdanova et al., 2001; Prober et al., 2006; Yokogawa et al., 2007). Furthermore, zebrafish larvae exhibit rest rebound and increased arousal thresholds following rest deprivation, another important criterion of sleep-like states (Zhdanova et al., 2001; Yokogawa et al., 2007). Second, mammalian anatomical and molecular aspects of sleep are conserved in zebrafish. In particular, the zebrafish hypocretin/orexin (Hcrt) system, a neuropeptide regulator of sleep and arousal in mammals, is conserved (Kaslin et al., 2004; Faraco et al., 2006; Prober et al., 2006; Yokogawa et al., 2007). Consistent with mammalian studies, overexpression of Hcrt in zebrafish dramatically decreases sleep (Prober et al., 2006), and mutations in the Hcrt receptor lead to sleep fragmentation at night (Yokogawa et al., 2007). Third, small molecules that modulate rest in mammals, including melatonin, benzodiazepines, barbiturates, histamine receptor $\mathrm{H} 1$ antagonists, and $\alpha-2$ adrenergic agonists have similar sedative effects in zebrafish larvae (Zhdanova et al., 2001; Ruuskanen et al., 2005; Renier et al., 2007). Together, these studies establish zebrafish as a good model system for the dissection of vertebrate sleep-like states through the use of both genetics and small molecules.

The short-term, small-scale pharmacological studies were extended by a long-term, highthroughput screen of the sleep/wake effects of nearly 4000 small molecules (Rihel et al., 
This is the pre-peer reviewed version of the following article: Rihel J and Schier AF (2012). Behavioral screening for neuroactive drugs in zebrafish. Developmental Neurobiology, 72(3):373-385., which has been published in final form at http://onlinelibrary.wiley.com/doi/10.1002/dneu.20910/full

2010a). Single larvae were placed into each of 80 wells of a 96 -well plate, and groups of ten larvae were exposed to a small molecule dissolved directly in the water (and $<0.3 \%$ DMSO) at four days post-fertilization. Automated tracking software then monitored the long term effects of each compound on multiple parameters of sleep/wake behavior, including the number and duration of sleep bouts, the length of sleep latency (i.e. the time from lights off/on to the first rest bout), and the average locomotor activity during both the day and the night (Figure $1 \mathrm{~A})$. Rihel et al. screened 5648 small molecules, representing nearly 4000 unique structures known to modulate a variety of biological functions and molecular pathways. Of these, more than 450 unique structures significantly altered sleep/wake behavior compared to DMSO-treated vehicle controls.

To help reduce the complexity of this large dataset, hierarchical clustering methods were used to organize the compounds by their multi-dimensional phenotypic output. These behavioral profiles (Figure 1A) organized compounds broadly into arousing and sedating compounds, but also identified compounds with selective effects on behavior. Behavioral profiles included increased locomotor activity only during the day or night, seizure-like responses, and effects on sleep latency. Notably, compounds that shared biological targets were more likely to cluster together, indicating that modulation of the same molecular pathway by diverse compounds produced similar phenotypic outputs. For example, selective serotonin reuptake inhibitors (SSRIs), including the structurally diverse 6-nitroquipazine, zimelidine, chlorpheniramine, and fluvoxamine, all increased total rest across the experiment. Given that these compounds are structurally diverse but have the same biological target further highlights the power of the dataset to group compounds specifically by molecular target and phenotype and not by virtue of their structural similarity. Moreover, agonists and antagonists often gave opposite phenotypic outcomes; for example, ß-adrenergic agonists (e.g. clenbuterol) increased waking activity while 
This is the pre-peer reviewed version of the following article: Rihel J and Schier AF (2012). Behavioral screening for neuroactive drugs in zebrafish. Developmental Neurobiology, 72(3):373-385., which has been published in final form at http://onlinelibrary.wiley.com/doi/10.1002/dneu.20910/full

ß-adrenergic antagonists (e.g. carvedilol) induced sedation. These observations further validated the links forged between the modulation of specific molecular pathway targets and behavioral outcomes, as opposed to non-specific and off-target effects.

A systematic analysis of behavior-altering compounds and their targets revealed a broad conservation of sleep/wake pharmacology between zebrafish and mammals and also revealed several modulated pathways that had been under- or unappreciated in sleep/wake biology. First, many modulators of neurotransmitter systems, including the noradrenaline, serotonin, dopamine, GABA, glutamate, histamine, adenosine, and melatonin systems, induced similar sleep/wake phenotypes in zebrafish as observed in mammals (Rihel et al., 2010a). Verapamillike L-type calcium channel inhibitors selectively increased rest with no effect on waking activity, ether-a-go-go related gene (ERG) potassium channel inhibitors selectively increased wakefulness at night, and a structural variety of anti-inflammatory compounds selectively increased daytime waking activity. Finally, Rihel et al. identified structurally related podocarpatrien-3-ones that specifically increased rest latency. The molecular pathway that these compounds modulate is currently unknown, but, given the clinical significance of sleep latency to insomnia (Culebras, 1996), modulation of this pathway could represent a novel therapeutic target for the treatment of sleep disorders. Clinical relevance aside, the small molecule screen was an effective way to rapidly expand the small molecule toolkit for future experiments in zebrafish sleep/wake biology and identify many potentially interesting targets to explore.

The clustering analysis not only identified small molecules that induce similar phenotypes but revealed that the targets of less-well characterized compounds could be predicted in a "guilt-byassociation" fashion by examining the cluster neighborhood of these poorly understood drugs. 
This is the pre-peer reviewed version of the following article: Rihel J and Schier AF (2012). Behavioral screening for neuroactive drugs in zebrafish. Developmental Neurobiology, 72(3):373-385., which has been published in final form at http://onlinelibrary.wiley.com/doi/10.1002/dneu.20910/full

The use of multi-dimensional dataset clustering to identify the targets or functional outputs of related compounds had been previously explored in other contexts, especially as applied to small molecule perturbation of gene expression (assessed by microarray). One pioneering study constructed a Connectivity Map and made predictions about drug targets by using gene expression analysis of cell culture lines exposed to small molecule perturbagens (Lamb et al., 2006). Other studies have made small molecule target predictions based on other gene expression datasets (Mori et al., 2009), anti-malarial mechanisms of action (Plouffe et al., 2008), and human side effect profiles (Campillos et al., 2008). Inspired by these studies, Rihel et al. used the behavioral profiles to make predictions about the targets of poorly characterized small molecules. For example, the adenosine A3 antagonist, MRS-1220, co-clustered with monoamine oxidase (MAO) inhibitors and turned out to inhibit MAO in vitro (Rihel et al., 2010a).

\section{Photomotor Response (PMR) Screen}

Although zebrafish larvae do not exhibit robust sleep/wake behaviors until five days post fertilization, they do exhibit many complex responses to various stimuli as early as 24 hours post fertilization. Seeking a reproducible, developmentally early, and short duration embryonic behavior to further enhance screening throughput, Kokel and colleagues discovered that in response to an intense light stimulus embryonic zebrafish exhibit a stereotypical motor behavior called the photomotor response (PMR; Figure 1B), (Kokel et al., 2010). During the background phase very few spontaneous movements are observed. A high-intensity light pulse elicits, after a latency phase of about one second, a prolonged (five to seven seconds) excitation phase, during which the larvae move vigorously. Following the excitation phase, larvae are refractory to additional light pulses for an extended time, and baseline activity is even lower than during the background phase. Initial tests of select psychoactive compounds, including stimulants and anxiolytics, altered the PMR behavioral output. Thus, while the behavior is relatively simple, it is 
This is the pre-peer reviewed version of the following article: Rihel J and Schier AF (2012). Behavioral screening for neuroactive drugs in zebrafish. Developmental Neurobiology, 72(3):373-385., which has been published in final form at http://onlinelibrary.wiley.com/doi/10.1002/dneu.20910/full

nevertheless under the control of multiple neurotransmitter systems and suitable for neuroactive small molecule screening.

Using an automated stage to deliver the light flash and tracking software to automatically observe behavioral responses in a 96-well plate format, Kokel and colleagues screened the effects of 14,000 small molecules on the PMR response and identified 982 unique structures that altered specific aspects of the PMR. This approach identified compounds that broadly increased activity (e.g. the stimulant isoproterenol), broadly increased sedation (e.g. the anxiolytic diazepam), increased response latency (e.g. the dopamine agonist apomorphine), or altered the refractory period (e.g. the SSRI 6-nitroquipazine) (Kokel et al., 2010). To organize this complex dataset, they further divided the PMR response into 14 behavioral parameters to generate a multi-dimensional profile that represents the behavioral phenotype elicited by each compound. They then used hierarchical clustering to organize small molecules by profile and examined the behavioral associations among compounds (Figure 1B). Importantly, the phenoclusters were often enriched with compounds that share mechanism of action. For example, Kokel et al. identified a hyperactive phenocluster of $ß$-adenergic receptor agonists, a latency phenocluster of dopamine agonists, and a dampened excitation phenocluster enriched for adenosine receptor antagonists.

The PMR clustergram was used to identify the molecular targets for novel compounds using the "guilt by association" method. One "slow to relax" phenocluster (characterized by prolonged tail flexion) was enriched for known acetylcholinesterase (AchE) inhibitors but also contained several uncharacterized and structurally unrelated compounds (named STR-1 and STR-2; "slow-to relax" = STR). Predicting that STR-1 and STR-2 were AchE inhibitors, Kokel et al. tested each in vitro and identified STR-1 as a bona fide structurally novel AchE inhibitor. 
This is the pre-peer reviewed version of the following article: Rihel J and Schier AF (2012). Behavioral screening for neuroactive drugs in zebrafish. Developmental Neurobiology, 72(3):373-385., which has been published in final form at http://onlinelibrary.wiley.com/doi/10.1002/dneu.20910/full

Intriguingly, STR-2 did not have AchE blocking activity in vitro, but when tested in vivo using larval lysates, STR-2 was a strong inhibitor. STR-2 requires activation by an in vivo biological process, an important finding that highlights another advantage of in vivo, whole animal screens - a classical in vitro screen would likely have missed this compound. Conversely, compounds with known in vitro activity can have dramatically altered or additional activities in vivo that cannot be predicted in advance. Taken together with the successful small molecule target prediction in Rihel et al. (2010a), these results suggest that behavioral profiling will be useful to identify previously unknown activities of small molecules. Going forward, larger studies will be needed to determine how reliably this approach can successfully identify targets.

\section{Conceptual and Practical Lessons}

The two small molecule behavioral screens provide the proof of principle that zebrafish screens can be used to uncover novel or unexpected neuroactive molecules. However, in order for whole animal behavioral profiling to become a major component of psychotropic drug discovery, several important issues must be addressed. As we will discuss, for some issues, these initial studies already hint at solutions; other issues will need to be addressed by on-going and future experiments.

\section{$\underline{\text { Dose }}$}

All small molecule screens must deal with the issue of dose. In any sufficiently large cell-based or whole animal screen, compromises must be made-- there are simply too many variables in play that will affect the appropriate dose for any given compound. This especially involves questions of absorption, distribution, metabolism, excretion, and toxicity (ADMET), which can be different for each small molecule. Ideally, with sufficiently high throughput (e.g. the rapid PMR screen), multiple doses for each compound can be tested. For both the sleep/wake and the PMR screens, pilot experiments tested known psychoactive compounds across a range of 
This is the pre-peer reviewed version of the following article: Rihel J and Schier AF (2012). Behavioral screening for neuroactive drugs in zebrafish. Developmental Neurobiology, 72(3):373-385., which has been published in final form at http://onlinelibrary.wiley.com/doi/10.1002/dneu.20910/full

concentrations; typically, high doses $(>30-100 \mu \mathrm{M})$ were toxic while low doses $(<300 \mathrm{nM})$ elicited no behavioral effects (Kokel et al., 2010; Rihel et al., 2010a). Importantly, within the effective concentration range, the behavioral profiles were relatively stable for most compounds, suggesting that similar phenotypic outputs will be identified for a range of doses. Inevitably, both screens contain false negatives that arise either from high-dose toxicity or low-dose ineffectiveness.

\section{Blood-Brain Barrier}

One issue related to dose is how the zebrafish blood-brain barrier may affect the ability of the screening compounds to reach the zebrafish brain. The blood-brain barrier is a protective layer of endothelial cells connected by tight junctions that can prevent transfer of molecules from the blood to the brain (Eliceiri et al., 2011). Recent evidence based on dye injections and expression of the tight junction proteins Zonula Occludens- 1 and Claudin-1 indicates that the zebrafish blood-brain barrier begins to develop at 3 days post fertilization (Jeong et al., 2008; Xie et al., 2010; Zhang et al., 2010). What this means for the bio-availability of small molecules (opposed to larger dye molecules) is unknown, especially since the circumventricular organ vessels lack a barrier at this age and leakage of vessels was still observed as late as 9 days post fertilization (Jeong et al., 2008; Xie et al., 2010). The initial behavioral screens were able to identify psychotropic compounds even at five to seven days post fertilization, indicating their ability to reach molecular targets within the larval zebrafish brain, but it is unknown what fraction of small compounds does not reach the brain.

\section{Poly-pharmacology}

One challenge to the discovery of novel neuroactive compounds for the treatment of CNS disorders is poly-pharmacology. Psychotropic drugs often affect multiple targets, and this broad 
This is the pre-peer reviewed version of the following article: Rihel J and Schier AF (2012). Behavioral screening for neuroactive drugs in zebrafish. Developmental Neurobiology, 72(3):373-385., which has been published in final form at http://onlinelibrary.wiley.com/doi/10.1002/dneu.20910/full

pharmacological activity may underlie the efficacy of many CNS drugs (Agid et al., 2007; Hopkins, 2008). The nature of single-target, in vitro screens makes it impossible to identify therapeutic compounds whose mechanism of action requires activity on multiple biological pathways. Whole animal behavioral screens, however, can uncover these promiscuous small molecules because the endpoint is a behavioral output that is dependent on the network interaction of multiple pathways. For example, in the sleep/wake screen, compounds with inhibitory activity on both dopamine reuptake and muscarinic acetylcholine receptors generated behavioral profiles distinct from small molecules that inhibited only one of these targets (Rihel et al., 2010a). Thus, anomalous phenoclusters within the behavioral dataset may motivate a deeper investigation of potential poly-pharmacological effects.

\section{Mapping of Small Molecules to Zebrafish Receptors}

The interpretation of zebrafish small molecule assays often rests on the assumption that the annotated activity of a small molecule, which is usually based on mammalian receptor data, will accurately reflect the activity on the zebrafish target. This need not be the case, however, as there can be dramatic inter-species activity differences, even among mammals. For example, the pharmacological profile of histamine receptors varies so widely between species that some compounds (e.g. FUB-322) are agonists on human histamine $\mathrm{H} 3$ receptors and inverse agonists on the rat receptor (Lovenberg et al., 2000; Seifert et al., 2003; Esbenshade et al., 2007). Discrepancies between zebrafish and mammalian receptor pharmacology could account for disparities in observed sleep/wake phenotypes in the small molecule screen. For example, dopamine D1 receptor agonists give opposite phenotypes in larval fish (sedation) and mammals (arousal) (Ongini et al., 1987; Bo et al., 1988; Trampus et al., 1993; Rihel et al., 2010a). It remains an open question whether this difference is due to altered activity of molecules on dopamine receptors or other differences in zebrafish dopamine system biology. The situation is 
This is the pre-peer reviewed version of the following article: Rihel J and Schier AF (2012). Behavioral screening for neuroactive drugs in zebrafish. Developmental Neurobiology, 72(3):373-385., which has been published in final form at http://onlinelibrary.wiley.com/doi/10.1002/dneu.20910/full

further complicated because of differences between the number of neurotransmitter receptor subtypes between humans and zebrafish. To take one example, there are five dopamine receptors in mammals, while there are eight in zebrafish (Panula et al., 2010). How small molecule activity maps to each of the zebrafish receptors in most cases is unknown.

Both the sleep/wake and the PMR screen partially mitigate the zebrafish annotation problem. First, confidence in the appropriate molecular interpretation is bolstered when multiple structurally diverse compounds give the same phenotype, especially when the small molecules target different components of the same signaling pathway. Second, the presence of agonist/antagonist pairs that yield opposite phenotypes is encouraging. Nevertheless, the systematic analysis of the binding activities of small molecules on zebrafish receptor subtypes will be needed to enhance the quality of drug library annotation for zebrafish small molecule screens. Such efforts include recent in vitro analyses that show conserved pharmacology of zebrafish opioid receptors (Gonzalez-Nunez et al., 2007; de Velasco et al., 2009), the nociceptin receptor (Rivas-Boyero et al., 2011), the zebrafish M2 muscarinic receptor (Hsieh and Liao, 2002), melanocortin receptors (Ringholm et al., 2002), the androgen receptor (Hossain et al., 2008) and bradykinnin receptors (Duner et al., 2002; Bromee et al., 2005). Radiolabel-binding studies of small molecule agonists/antagonists in zebrafish brain membranes or slices are also useful, especially when coupled with competitive binding assays, as shown in studies on muscarinic acetylcholine receptors (Williams and Messer, 2004), GABA-A and GABA-B receptors (Renier et al., 2007), H2 and H3 histamine receptors (Peitsaro et al., 2000; Peitsaro et al., 2007), and adrenergic receptors (Ruuskanen et al., 2005). While these studies paint an overall picture of conservation between zebrafish and mammalian neural signaling systems, more detailed characterization will greatly assist the proper interpretation of small molecule behavioral screening efforts. 
This is the pre-peer reviewed version of the following article: Rihel J and Schier AF (2012). Behavioral screening for neuroactive drugs in zebrafish. Developmental Neurobiology, 72(3):373-385., which has been published in final form at http://onlinelibrary.wiley.com/doi/10.1002/dneu.20910/full

\section{Expansion of the Behavioral Assays}

In principle, as long as a behavior is modulated by multiple major neuronal signaling systems, that behavior can be used to discover novel psychotropic compounds. However, this approach effectively limits the scope of the neuroactive screen to only those molecular signaling pathways involved in the screened behavior, leaving potentially important systems unexplored. One way to combat this issue is to systematically increase the behavioral search space and expand the multidimensional behavioral profile to include many more behavioral parameters. There is an ever-expanding list of larval zebrafish behaviors that are amenable to high-throughput screens and that can be included as part of an automated behavioral screening repertoire (Kokel and Peterson, 2008; Mathur and Guo, 2010; Tierney, 2011). These include a range of visual behaviors such as eye saccade responses to a rotating drum (the optokinetic reflex, or OKR) (Brockerhoff et al., 1995), motor responses to a moving grating (optomotor response, or OMR) (Neuhauss et al., 1999), or locomotor responses to changes in light intensity (the visual-motor response, VMR) (Emran et al., 2007; Emran et al., 2010). Zebrafish larvae also have complex behavioral startle responses to acoustic stimuli (Burgess and Granato, 2007), and these responses habituate over time as a form of non-associative learning (Best et al., 2008). Additionally, the zebrafish startle response to a high intensity stimulus can be inhibited by a lower intensity pulse (pre-pulse inhibition, PPI) (Burgess and Granato, 2007). Since change in PPI is an endophenotype associated with human schizophrenia (Braff et al., 2001), the zebrafish acoustic startle and PPI phenotypes are an exciting assay for further study and have already yielded a PPI impaired mutant, Ophelia (Burgess and Granato, 2007). Larval zebrafish also exhibit locomotor responses to changes in temperature and noxious chemicals (Prober et al., 2008). Finally, assays to study more integrated behaviors, such as prey capture, are also established in zebrafish larvae (Budick and O'Malley, 2000; Borla et al., 2002; Gahtan et al., 
This is the pre-peer reviewed version of the following article: Rihel J and Schier AF (2012). Behavioral screening for neuroactive drugs in zebrafish. Developmental Neurobiology, 72(3):373-385., which has been published in final form at http://onlinelibrary.wiley.com/doi/10.1002/dneu.20910/full

2005). Combining several of these behavioral responses together into a single assay to generate a larger behavioral profile will be an attractive way to increase the resolving power and the search space of neuroactive screens.

\section{Clinical Relevance of Behavioral Assays}

Although direct human clinical relevance is not essential for fruitful neuroactive screening, disease-relevant behavioral models are also important, especially to identify the most promising candidate molecules for mammalian follow-up studies. No animal model can perfectly recapitulate complex human psychiatric and CNS diseases, but the closer an animal model can mimic critical aspects of disease, the more likely the search for disease-ameliorating small molecules will be successful. One way to create more disease relevant zebrafish models is to identify small molecule pre-treatments that recapitulate important clinical aspects of disease. For example, the sleep/wake screen found that MK-801 and other NMDA receptor antagonists used in drug-induced schizophrenia animal models (Olney et al., 1999) similarly increased locomotor behavior (Adriani et al., 1998; Rihel et al., 2010a), a result confirmed in other adult and larval zebrafish experiments (Swain et al., 2004; Chen et al., 2010; Seibt et al., 2010). The PMR screen identified paralysis in zebrafish treated with organophosphates (Kokel et al., 2010), a phenotype that was reversed by antidotes used to treat human organophosphate toxicity (Bajgar, 2004). Pentylenetetrazole (PTZ) treatment, which causes seizures in zebrafish larvae (Baraban et al., 2005), was used to identify potential seizure resistance genes (Baraban et al., 2007) and would be amenable to small molecule screens for novel anti-convulsants. Other drug-induced larval phenotypes with promising clinical relevance include ethanol intoxication (Lockwood et al., 2004; MacPhail et al., 2009), nicotine dependence (Petzold et al., 2009), and 1-methyl-4-phenyl-1,2,3,6-tetrahydropyridine (MPTP) induced ablation of dopamine neurons as a model for Parkinson's disease (Bretaud et al., 2004; Lam et al., 2005; McKinley et al., 2005; 
This is the pre-peer reviewed version of the following article: Rihel J and Schier AF (2012). Behavioral screening for neuroactive drugs in zebrafish. Developmental Neurobiology, 72(3):373-385., which has been published in final form at http://onlinelibrary.wiley.com/doi/10.1002/dneu.20910/full

Thirumalai and Cline, 2008). Finally, zebrafish mutant models of human psychiatric diseases can serve as chemical screening start points. The development of relevant zebrafish behavioral and CNS mutants is still in its infancy but rapidly expanding to include neurodegenerative disorders, autism, schizophrenia, and Huntington's disease (see recent reviews by Best and Alderton, 2008; Kabashi et al., 2010; Mathur and Guo, 2010; Tierney, 2011). Small molecule suppressor/enhancer screens on the relevant mutant backgrounds will be a rapid way to identify molecular pathways that interact with disease genes.

\section{Conclusions}

Small molecule behavioral screening can now be performed with sufficient throughput to be useful in the discovery of neuroactive compounds. Where might such zebrafish screens fit in the neuroactive drug pipeline? They could be used as a testing ground for previously curated small molecule leads at a stage before more expensive testing is performed in rodents. For example, much effort has recently been put into the identification of potent hypocretin receptor antagonists such as almorexant (Brisbare-Roch et al., 2007; Neubauer, 2010). Screening candidate compounds in zebrafish behavioral models of hypocretin-induced hyperactivity (Prober et al., 2006) could serve as a cheap way to identify the most promising non-toxic molecules with good in vivo pharmacological activity before testing in mammals. As even higher throughput is achieved, zebrafish screens could also serve as a primary and early screening stage for novel neuroactives that would then be further optimized for increased potency by medicinal chemists. By testing a larger and more diverse set of chemical structures with unknown pharmacological properties, zebrafish behavioral assays could identify wholly novel neuroactive compounds. Unlike in vitro assays designed around single targets, these newly identified compounds would already be shown to have promising in vivo properties. 
This is the pre-peer reviewed version of the following article: Rihel J and Schier AF (2012). Behavioral screening for neuroactive drugs in zebrafish. Developmental Neurobiology, 72(3):373-385., which has been published in final form at http://onlinelibrary.wiley.com/doi/10.1002/dneu.20910/full

Finally, one should not ignore another powerful use of the small molecule screening paradigmto gain novel insights in the mechanistic underpinnings of behavior. Using well annotated chemical libraries, these molecules become tools to rapidly test the functional importance of many neuro-modulatory pathways in specific behaviors. Once critical neural signaling events have been implicated as modulators of behavior by pathway agonists and antagonists, more targeted experiments can be designed to more deeply test how the pathway modulates behavior and where in the brain that modulation takes place. Thus, the small molecule screens provide a bridge between molecular targets and behavioral outputs. A future challenge will be to carefully dissect the underlying neuronal circuits that underpin this behavioral control.

Figure 1. Converting Quantitative Behavioral Data into Behavioral Profiles for Clustering. A) In the sleep/wake screen, larval activity data is collected over several days (left). The data for each small molecule gets broken into multiple parameters and converted into a behavioral profile for hierarchical clustering (right). The clustering organizes the drugs (rows) based on phenotype. In this example, the top three drugs cluster because they increase average sleep bout lengths. The lower cluster contains three drugs that increase daytime waking activity. B) In the PMR screen, 30 seconds of larval activity data in response to an intense light pulse is collected (left). This data is also converted into behavioral profiles and clustered based on phenotype (right). In the top cluster, three drugs increase activity during the refractory period, whereas in the lower cluster, three drugs shorten the latency period. Modified from Rihel et al., (2010) and Kokel et al., (2010).

\section{References}


This is the pre-peer reviewed version of the following article: Rihel J and Schier AF (2012). Behavioral screening for neuroactive drugs in zebrafish. Developmental Neurobiology, 72(3):373-385., which has been published in final form at http://onlinelibrary.wiley.com/doi/10.1002/dneu.20910/full

Adriani, W., Felici, A., Sargolini, F., Roullet, P., Usiello, A., Oliverio, A., and Mele, A. (1998). Nmethyl-D-aspartate and dopamine receptor involvement in the modulation of locomotor activity and memory processes. Exp Brain Res 123, 52-59.

Agid, Y., Buzsaki, G., Diamond, D.M., Frackowiak, R., Giedd, J., Girault, J.A., Grace, A., Lambert, J.J., Manji, H., Mayberg, H., et al. (2007). How can drug discovery for psychiatric disorders be improved? Nat Rev Drug Discov 6, 189-201.

Appelbaum, L., Wang, G.X., Maro, G.S., Mori, R., Tovin, A., Marin, W., Yokogawa, T., Kawakami, K., Smith, S.J., Gothilf, Y., et al. (2009). Sleep-wake regulation and hypocretinmelatonin interaction in zebrafish. Proc Natl Acad Sci U S A 106, 21942-21947.

Bajgar, J. (2004). Organophosphates/nerve agent poisoning: mechanism of action, diagnosis, prophylaxis, and treatment. Adv Clin Chem 38, 151-216.

Ban, T.A. (2006). The role of serendipity in drug discovery. Dialogues Clin Neurosci 8, 335-344.

Baraban, S.C., Dinday, M.T., Castro, P.A., Chege, S., Guyenet, S., and Taylor, M.R. (2007). A large-scale mutagenesis screen to identify seizure-resistant zebrafish. Epilepsia 48, 1151-1157.

Baraban, S.C., Taylor, M.R., Castro, P.A., and Baier, H. (2005). Pentylenetetrazole induced changes in zebrafish behavior, neural activity and c-fos expression. Neuroscience 131, 759768.

Bencan, Z., and Levin, E.D. (2008). The role of alpha7 and alpha4beta2 nicotinic receptors in the nicotine-induced anxiolytic effect in zebrafish. Physiol Behav 95, 408-412.

Bencan, Z., Sledge, D., and Levin, E.D. (2009). Buspirone, chlordiazepoxide and diazepam effects in a zebrafish model of anxiety. Pharmacol Biochem Behav 94, 75-80.

Best, J.D., and Alderton, W.K. (2008). Zebrafish: An in vivo model for the study of neurological diseases. Neuropsychiatr Dis Treat 4, 567-576.

Best, J.D., Berghmans, S., Hunt, J.J., Clarke, S.C., Fleming, A., Goldsmith, P., and Roach, A.G. (2008). Non-associative learning in larval zebrafish. Neuropsychopharmacology 33, 1206-1215.

Bilotta, J., Saszik, S., Givin, C.M., Hardesty, H.R., and Sutherland, S.E. (2002). Effects of embryonic exposure to ethanol on zebrafish visual function. Neurotoxicol Teratol 24, 759-766.

Blank, M., Guerim, L.D., Cordeiro, R.F., and Vianna, M.R. (2009). A one-trial inhibitory avoidance task to zebrafish: rapid acquisition of an NMDA-dependent long-term memory. Neurobiol Learn Mem 92, 529-534.

Blaser, R.E., Koid, A., and Poliner, R.M. (2010). Context-dependent sensitization to ethanol in zebrafish (Danio rerio). Pharmacol Biochem Behav 95, 278-284.

Bo, P., Ongini, E., Giorgetti, A., and Savoldi, F. (1988). Synchronization of the EEG and sedation induced by neuroleptics depend upon blockade of both D1 and D2 dopamine receptors. Neuropharmacology 27, 799-805. 
This is the pre-peer reviewed version of the following article: Rihel J and Schier AF (2012). Behavioral screening for neuroactive drugs in zebrafish. Developmental Neurobiology, 72(3):373-385., which has been published in final form at http://onlinelibrary.wiley.com/doi/10.1002/dneu.20910/full

Boehmler, W., Carr, T., Thisse, C., Thisse, B., Canfield, V.A., and Levenson, R. (2007). D4 Dopamine receptor genes of zebrafish and effects of the antipsychotic clozapine on larval swimming behaviour. Genes Brain Behav 6, 155-166.

Borla, M.A., Palecek, B., Budick, S., and O'Malley, D.M. (2002). Prey capture by larval zebrafish: evidence for fine axial motor control. Brain Behav Evol 60, 207-229.

Braff, D.L., Geyer, M.A., Light, G.A., Sprock, J., Perry, W., Cadenhead, K.S., and Swerdlow, N.R. (2001). Impact of prepulse characteristics on the detection of sensorimotor gating deficits in schizophrenia. Schizophr Res 49,171-178.

Bretaud, S., Lee, S., and Guo, S. (2004). Sensitivity of zebrafish to environmental toxins implicated in Parkinson's disease. Neurotoxicol Teratol 26, 857-864.

Brisbare-Roch, C., Dingemanse, J., Koberstein, R., Hoever, P., Aissaoui, H., Flores, S., Mueller, C., Nayler, O., van Gerven, J., de Haas, S.L., et al. (2007). Promotion of sleep by targeting the orexin system in rats, dogs and humans. Nat Med 13, 150-155.

Brockerhoff, S.E., Hurley, J.B., Janssen-Bienhold, U., Neuhauss, S.C., Driever, W., and Dowling, J.E. (1995). A behavioral screen for isolating zebrafish mutants with visual system defects. Proc Natl Acad Sci U S A 92, 10545-10549.

Bromee, T., Kukkonen, J.P., Andersson, P., Conlon, J.M., and Larhammar, D. (2005). Pharmacological characterization of ligand-receptor interactions at the zebrafish bradykinin receptor. Br J Pharmacol 144, 11-16.

Budi, E.H., Patterson, L.B., and Parichy, D.M. (2008). Embryonic requirements for ErbB signaling in neural crest development and adult pigment pattern formation. Development 135, 2603-2614.

Budick, S.A., and O'Malley, D.M. (2000). Locomotor repertoire of the larval zebrafish: swimming, turning and prey capture. J Exp Biol 203, 2565-2579.

Burgess, H.A., and Granato, M. (2007). Sensorimotor gating in larval zebrafish. J Neurosci 27, 4984-4994.

Cachat, J., Canavello, P., Elegante, M., Bartels, B., Hart, P., Bergner, C., Egan, R., Duncan, A., Tien, D., Chung, A., et al. (2010). Modeling withdrawal syndrome in zebrafish. Behav Brain Res 208, 371-376.

Campillos, M., Kuhn, M., Gavin, A.C., Jensen, L.J., and Bork, P. (2008). Drug target identification using side-effect similarity. Science 321, 263-266.

Chen, J., Patel, R., Friedman, T.C., and Jones, K.S. (2010). The Behavioral and Pharmacological Actions of NMDA Receptor Antagonism are Conserved in Zebrafish Larvae. Int J Comp Psychol 23, 82-90.

Clifton, J.D., Lucumi, E., Myers, M.C., Napper, A., Hama, K., Farber, S.A., Smith, A.B., 3rd, Huryn, D.M., Diamond, S.L., and Pack, M. (2010). Identification of novel inhibitors of dietary lipid absorption using zebrafish. PLoS One 5, e12386. 
This is the pre-peer reviewed version of the following article: Rihel J and Schier AF (2012). Behavioral screening for neuroactive drugs in zebrafish. Developmental Neurobiology, 72(3):373-385., which has been published in final form at http://onlinelibrary.wiley.com/doi/10.1002/dneu.20910/full

Culebras, A. (1996). Clinical handbook of sleep disorders (Boston, Butterworth-Heinemann).

Darland, T., and Dowling, J.E. (2001). Behavioral screening for cocaine sensitivity in mutagenized zebrafish. Proc Natl Acad Sci U S A 98, 11691-11696.

de Velasco, E.M., Law, P.Y., and Rodriguez, R.E. (2009). Mu opioid receptor from the zebrafish exhibits functional characteristics as those of mammalian mu opioid receptor. Zebrafish 6, 259268.

Dlugos, C.A., Brown, S.J., and Rabin, R.A. (2011). Gender differences in ethanol-induced behavioral sensitivity in zebrafish. Alcohol 45, 11-18.

Dlugos, C.A., and Rabin, R.A. (2003). Ethanol effects on three strains of zebrafish: model system for genetic investigations. Pharmacol Biochem Behav 74, 471-480.

Duner, T., Conlon, J.M., Kukkonen, J.P., Akerman, K.E., Yan, Y.L., Postlethwait, J.H., and Larhammar, D. (2002). Cloning, structural characterization and functional expression of a zebrafish bradykinin B2-related receptor. Biochem J 364, 817-824.

Egan, R.J., Bergner, C.L., Hart, P.C., Cachat, J.M., Canavello, P.R., Elegante, M.F., Elkhayat, S.I., Bartels, B.K., Tien, A.K., Tien, D.H., et al. (2009). Understanding behavioral and physiological phenotypes of stress and anxiety in zebrafish. Behav Brain Res 205, 38-44.

Eliceiri, B.P., Gonzalez, A.M., and Baird, A. (2011). Zebrafish model of the blood-brain barrier: morphological and permeability studies. Methods Mol Biol 686, 371-378.

Emran, F., Rihel, J., Adolph, A.R., Wong, K.Y., Kraves, S., and Dowling, J.E. (2007). OFF ganglion cells cannot drive the optokinetic reflex in zebrafish. Proc Natl Acad Sci U S A 104, 19126-19131.

Emran, F., Rihel, J., Adolph, A.R., and Dowling, J.E. (2010). Larval zebrafish lose vision at night. Proc Natl Acad Sci U S A 107, 6034-6039

Esbenshade, T.A., Estvander, B.R., Miller, T.R., Baranowski, J.L., Sharma, R., Hancock, A.A., and Krueger, K.M. (2007). Pharmacological classification of histamine H3 receptor agents across species is attributable to transmembrane 3 sequence differences. Inflamm Res 56 Suppl $1, \mathrm{~S} 45-46$.

Faraco, J.H., Appelbaum, L., Marin, W., Gaus, S.E., Mourrain, P., and Mignot, E. (2006). Regulation of hypocretin (orexin) expression in embryonic zebrafish. J Biol Chem 281, 2975329761.

Fernandes, Y., and Gerlai, R. (2009). Long-term behavioral changes in response to early developmental exposure to ethanol in zebrafish. Alcohol Clin Exp Res 33, 601-609.

Fischer-Barnicol, D., Lanquillon, S., Haen, E., Zofel, P., Koch, H.J., Dose, M., and Klein, H.E. (2008). Typical and atypical antipsychotics--the misleading dichotomy. Results from the Working Group 'Drugs in Psychiatry' (AGATE). Neuropsychobiology 57, 80-87.

Gahtan, E., Tanger, P., and Baier, H. (2005). Visual prey capture in larval zebrafish is controlled by identified reticulospinal neurons downstream of the tectum. J Neurosci 25, 9294-9303. 
This is the pre-peer reviewed version of the following article: Rihel J and Schier AF (2012). Behavioral screening for neuroactive drugs in zebrafish. Developmental Neurobiology, 72(3):373-385., which has been published in final form at http://onlinelibrary.wiley.com/doi/10.1002/dneu.20910/full

Geddes, J., Freemantle, N., Harrison, P., and Bebbington, P. (2000). Atypical antipsychotics in the treatment of schizophrenia: systematic overview and meta-regression analysis. BMJ 321, 1371-1376.

Gerlai, R., Ahmad, F., and Prajapati, S. (2008). Differences in acute alcohol-induced behavioral responses among zebrafish populations. Alcohol Clin Exp Res 32, 1763-1773.

Gerlai, R., Chatterjee, D., Pereira, T., Sawashima, T., and Krishnannair, R. (2009). Acute and chronic alcohol dose: population differences in behavior and neurochemistry of zebrafish. Genes Brain Behav 8, 586-599.

Gerlai, R., Lahav, M., Guo, S., and Rosenthal, A. (2000). Drinks like a fish: zebra fish (Danio rerio) as a behavior genetic model to study alcohol effects. Pharmacol Biochem Behav 67, 773782.

Gerlai, R., Lee, V., and Blaser, R. (2006). Effects of acute and chronic ethanol exposure on the behavior of adult zebrafish (Danio rerio). Pharmacol Biochem Behav 85, 752-761.

Giacomini, N.J., Rose, B., Kobayashi, K., and Guo, S. (2006). Antipsychotics produce locomotor impairment in larval zebrafish. Neurotoxicol Teratol 28, 245-250.

Gonzalez-Nunez, V., Marron Fernandez de Velasco, E., Arsequell, G., Valencia, G., and Rodriguez, R.E. (2007). Identification of dynorphin a from zebrafish: a comparative study with mammalian dynorphin A. Neuroscience 144, 675-684.

Grossman, L., Utterback, E., Stewart, A., Gaikwad, S., Chung, K.M., Suciu, C., Wong, K., Elegante, M., Elkhayat, S., Tan, J., et al. (2010). Characterization of behavioral and endocrine effects of LSD on zebrafish. Behav Brain Res 214, 277-284.

Hirayama, J., Kaneko, M., Cardone, L., Cahill, G., and Sassone-Corsi, P. (2005). Analysis of circadian rhythms in zebrafish. Methods Enzymol 393, 186-204.

Hopkins, A.L. (2008). Network pharmacology: the next paradigm in drug discovery. Nat Chem Biol 4, 682-690.

Hossain, M.S., Larsson, A., Scherbak, N., Olsson, P.E., and Orban, L. (2008). Zebrafish androgen receptor: isolation, molecular, and biochemical characterization. Biol Reprod 78, 361369.

Hsieh, D.J., and Liao, C.F. (2002). Zebrafish M2 muscarinic acetylcholine receptor: cloning, pharmacological characterization, expression patterns and roles in embryonic bradycardia. $\mathrm{Br} \mathrm{J}$ Pharmacol 137, 782-792.

Hultman, K.A., Scott, A.W., and Johnson, S.L. (2008). Small molecule modifier screen for kitdependent functions in zebrafish embryonic melanocytes. Zebrafish 5, 279-287.

Irons, T.D., MacPhail, R.C., Hunter, D.L., and Padilla, S. (2010). Acute neuroactive drug exposures alter locomotor activity in larval zebrafish. Neurotoxicol Teratol 32, 84-90.

Jeong, J.Y., Kwon, H.B., Ahn, J.C., Kang, D., Kwon, S.H., Park, J.A., and Kim, K.W. (2008). Functional and developmental analysis of the blood-brain barrier in zebrafish. Brain Res Bull 75, 619-628. 
This is the pre-peer reviewed version of the following article: Rihel J and Schier AF (2012). Behavioral screening for neuroactive drugs in zebrafish. Developmental Neurobiology, 72(3):373-385., which has been published in final form at http://onlinelibrary.wiley.com/doi/10.1002/dneu.20910/full

Kabashi, E., Champagne, N., Brustein, E., and Drapeau, P. (2010). In the swim of things: recent insights to neurogenetic disorders from zebrafish. Trends Genet 26, 373-381.

Kaslin, J., Nystedt, J.M., Ostergard, M., Peitsaro, N., and Panula, P. (2004). The orexin/hypocretin system in zebrafish is connected to the aminergic and cholinergic systems. $J$ Neurosci 24, 2678-2689.

Kily, L.J., Cowe, Y.C., Hussain, O., Patel, S., McElwaine, S., Cotter, F.E., and Brennan, C.H. (2008). Gene expression changes in a zebrafish model of drug dependency suggest conservation of neuro-adaptation pathways. J Exp Biol 211, 1623-1634.

Kim, Y.H., Lee, Y., Kim, D., Jung, M.W., and Lee, C.J. (2010). Scopolamine-induced learning impairment reversed by physostigmine in zebrafish. Neurosci Res 67, 156-161.

Kitambi, S.S., McCulloch, K.J., Peterson, R.T., and Malicki, J.J. (2009). Small molecule screen for compounds that affect vascular development in the zebrafish retina. Mech Dev 126, 464477.

Kokel, D., Bryan, J., Laggner, C., White, R., Cheung, C.Y., Mateus, R., Healey, D., Kim, S., Werdich, A.A., Haggarty, S.J., et al. (2010). Rapid behavior-based identification of neuroactive small molecules in the zebrafish. Nat Chem Biol 6, 231-237.

Kokel, D., and Peterson, R.T. (2008). Chemobehavioural phenomics and behaviour-based psychiatric drug discovery in the zebrafish. Brief Funct Genomic Proteomic 7, 483-490.

Lam, C.S., Korzh, V., and Strahle, U. (2005). Zebrafish embryos are susceptible to the dopaminergic neurotoxin MPTP. Eur J Neurosci 21, 1758-1762.

Lamb, J., Crawford, E.D., Peck, D., Modell, J.W., Blat, I.C., Wrobel, M.J., Lerner, J., Brunet, J.P., Subramanian, A., Ross, K.N., et al. (2006). The Connectivity Map: using gene-expression signatures to connect small molecules, genes, and disease. Science 313, 1929-1935.

Lau, B., Bretaud, S., Huang, Y., Lin, E., and Guo, S. (2006). Dissociation of food and opiate preference by a genetic mutation in zebrafish. Genes Brain Behav 5, 497-505.

Lau, B.Y., Mathur, P., Gould, G.G., and Guo, S. (2011). Identification of a brain center whose activity discriminates a choice behavior in zebrafish. Proc Natl Acad Sci U S A 108, 2581-2586.

Levin, E.D., Bencan, Z., and Cerutti, D.T. (2007). Anxiolytic effects of nicotine in zebrafish. Physiol Behav 90, 54-58.

Levin, E.D., and Chen, E. (2004). Nicotinic involvement in memory function in zebrafish. Neurotoxicol Teratol 26, 731-735.

Levin, E.D., Limpuangthip, J., Rachakonda, T., and Peterson, M. (2006). Timing of nicotine effects on learning in zebrafish. Psychopharmacology (Berl) 184, 547-552.

Lockwood, B., Bjerke, S., Kobayashi, K., and Guo, S. (2004). Acute effects of alcohol on larval zebrafish: a genetic system for large-scale screening. Pharmacol Biochem Behav 77, 647-654.

Lopez-Patino, M.A., Yu, L., Cabral, H., and Zhdanova, I.V. (2008). Anxiogenic effects of cocaine withdrawal in zebrafish. Physiol Behav 93, 160-171. 
This is the pre-peer reviewed version of the following article: Rihel J and Schier AF (2012). Behavioral screening for neuroactive drugs in zebrafish. Developmental Neurobiology, 72(3):373-385., which has been published in final form at http://onlinelibrary.wiley.com/doi/10.1002/dneu.20910/full

Lopez Patino, M.A., Yu, L., Yamamoto, B.K., and Zhdanova, I.V. (2008). Gender differences in zebrafish responses to cocaine withdrawal. Physiol Behav 95, 36-47.

Lord, A.M., North, T.E., and Zon, L.I. (2007). Prostaglandin E2: making more of your marrow. Cell Cycle 6, 3054-3057.

Lovenberg, T.W., Pyati, J., Chang, H., Wilson, S.J., and Erlander, M.G. (2000). Cloning of rat histamine $\mathrm{H}(3)$ receptor reveals distinct species pharmacological profiles. J Pharmacol Exp Ther 293, 771-778.

MacPhail, R.C., Brooks, J., Hunter, D.L., Padnos, B., Irons, T.D., and Padilla, S. (2009). Locomotion in larval zebrafish: Influence of time of day, lighting and ethanol. Neurotoxicology $30,52-58$.

MacRae, C.A., and Peterson, R.T. (2003). Zebrafish-based small molecule discovery. Chem Biol 10, 901-908.

Mathew, L.K., Sengupta, S., Kawakami, A., Andreasen, E.A., Lohr, C.V., Loynes, C.A., Renshaw, S.A., Peterson, R.T., and Tanguay, R.L. (2007). Unraveling tissue regeneration pathways using chemical genetics. J Biol Chem 282, 35202-35210.

Mathur, P., and Guo, S. (2010). Use of zebrafish as a model to understand mechanisms of addiction and complex neurobehavioral phenotypes. Neurobiol Dis 40, 66-72.

Mathur, P., and Guo, S. (2011). Differences of acute versus chronic ethanol exposure on anxiety-like behavioral responses in zebrafish. Behav Brain Res.

Maximino, C., da Silva, A.W., Gouveia, A., Jr., and Herculano, A.M. (2011). Pharmacological analysis of zebrafish (Danio rerio) scototaxis. Prog Neuropsychopharmacol Biol Psychiatry.

McKinley, E.T., Baranowski, T.C., Blavo, D.O., Cato, C., Doan, T.N., and Rubinstein, A.L. (2005). Neuroprotection of MPTP-induced toxicity in zebrafish dopaminergic neurons. Brain Res Mol Brain Res 141, 128-137.

Miller, G. (2010). Is pharma running out of brainy ideas? Science 329, 502-504.

Molina, G., Vogt, A., Bakan, A., Dai, W., Queiroz de Oliveira, P., Znosko, W., Smithgall, T.E., Bahar, I., Lazo, J.S., Day, B.W., et al. (2009). Zebrafish chemical screening reveals an inhibitor of Dusp6 that expands cardiac cell lineages. Nat Chem Biol 5, 680-687.

Mori, S., Chang, J.T., Andrechek, E.R., Potti, A., and Nevins, J.R. (2009). Utilization of genomic signatures to identify phenotype-specific drugs. PLoS One 4, e6772.

Murphey, R.D., Stern, H.M., Straub, C.T., and Zon, L.I. (2006). A chemical genetic screen for cell cycle inhibitors in zebrafish embryos. Chem Biol Drug Des 68, 213-219.

Neubauer, D.N. (2010). Almorexant, a dual orexin receptor antagonist for the treatment of insomnia. Curr Opin Investig Drugs 11, 101-110. 
This is the pre-peer reviewed version of the following article: Rihel J and Schier AF (2012). Behavioral screening for neuroactive drugs in zebrafish. Developmental Neurobiology, 72(3):373-385., which has been published in final form at http://onlinelibrary.wiley.com/doi/10.1002/dneu.20910/full

Neuhauss, S.C., Biehlmaier, O., Seeliger, M.W., Das, T., Kohler, K., Harris, W.A., and Baier, H. (1999). Genetic disorders of vision revealed by a behavioral screen of 400 essential loci in zebrafish. J Neurosci 19, 8603-8615.

North, T.E., Goessling, W., Peeters, M., Li, P., Ceol, C., Lord, A.M., Weber, G.J., Harris, J., Cutting, C.C., Huang, P., et al. (2009). Hematopoietic stem cell development is dependent on blood flow. Cell 137, 736-748.

North, T.E., Goessling, W., Walkley, C.R., Lengerke, C., Kopani, K.R., Lord, A.M., Weber, G.J., Bowman, T.V., Jang, I.H., Grosser, T., et al. (2007). Prostaglandin E2 regulates vertebrate haematopoietic stem cell homeostasis. Nature 447, 1007-1011.

Olney, J.W., Newcomer, J.W., and Farber, N.B. (1999). NMDA receptor hypofunction model of schizophrenia. J Psychiatr Res 33, 523-533.

Ongini, E., Caporali, M.G., and Longo, V.G. (1987). Blockade of D-1 dopamine receptors by SCH 23390 prevents EEG and behavioral activation induced by L-dopa. Neurosci Lett 82, 206210.

Oppedal, D., and Goldsmith, M.I. (2010). A chemical screen to identify novel inhibitors of fin regeneration in zebrafish. Zebrafish 7, 53-60.

Ou, H.C., Cunningham, L.L., Francis, S.P., Brandon, C.S., Simon, J.A., Raible, D.W., and Rubel, E.W. (2009). Identification of FDA-approved drugs and bioactives that protect hair cells in the zebrafish (Danio rerio) lateral line and mouse (Mus musculus) utricle. J Assoc Res Otolaryngol 10, 191-203.

Owens, K.N., Santos, F., Roberts, B., Linbo, T., Coffin, A.B., Knisely, A.J., Simon, J.A., Rubel, E.W., and Raible, D.W. (2008). Identification of genetic and chemical modulators of zebrafish mechanosensory hair cell death. PLoS Genet 4, e1000020.

Pangalos, M.N., Schechter, L.E., and Hurko, O. (2007). Drug development for CNS disorders: strategies for balancing risk and reducing attrition. Nat Rev Drug Discov 6, 521-532.

Panula, P., Chen, Y.C., Priyadarshini, M., Kudo, H., Semenova, S., Sundvik, M., and Sallinen, V. (2010). The comparative neuroanatomy and neurochemistry of zebrafish CNS systems of relevance to human neuropsychiatric diseases. Neurobiol Dis 40, 46-57.

Pardo-Martin, C., Chang, T.Y., Koo, B.K., Gilleland, C.L., Wasserman, S.C., and Yanik, M.F. (2010). High-throughput in vivo vertebrate screening. Nat Methods 7, 634-636.

Peal, D.S., Mills, R.W., Lynch, S.N., Mosley, J.M., Lim, E., Ellinor, P.T., January, C.T., Peterson, R.T., and Milan, D.J. (2011). Novel chemical suppressors of long QT syndrome identified by an in vivo functional screen. Circulation 123, 23-30.

Peal, D.S., Peterson, R.T., and Milan, D. (2010). Small molecule screening in zebrafish. J Cardiovasc Transl Res 3, 454-460.

Peitsaro, N., Anichtchik, O.V., and Panula, P. (2000). Identification of a histamine H(3)-like receptor in the zebrafish (Danio rerio) brain. J Neurochem 75, 718-724. 
This is the pre-peer reviewed version of the following article: Rihel J and Schier AF (2012). Behavioral screening for neuroactive drugs in zebrafish. Developmental Neurobiology, 72(3):373-385., which has been published in final form at http://onlinelibrary.wiley.com/doi/10.1002/dneu.20910/full

Peitsaro, N., Kaslin, J., Anichtchik, O.V., and Panula, P. (2003). Modulation of the histaminergic system and behaviour by alpha-fluoromethylhistidine in zebrafish. J Neurochem 86, 432-441.

Peitsaro, N., Sundvik, M., Anichtchik, O.V., Kaslin, J., and Panula, P. (2007). Identification of zebrafish histamine $\mathrm{H} 1, \mathrm{H} 2$ and $\mathrm{H} 3$ receptors and effects of histaminergic ligands on behavior. Biochem Pharmacol 73, 1205-1214.

Peterson, R.T., Link, B.A., Dowling, J.E., and Schreiber, S.L. (2000). Small molecule developmental screens reveal the logic and timing of vertebrate development. Proc Natl Acad Sci U S A 97, 12965-12969.

Peterson, R.T., Shaw, S.Y., Peterson, T.A., Milan, D.J., Zhong, T.P., Schreiber, S.L., MacRae, C.A., and Fishman, M.C. (2004). Chemical suppression of a genetic mutation in a zebrafish model of aortic coarctation. Nat Biotechnol 22, 595-599.

Petzold, A.M., Balciunas, D., Sivasubbu, S., Clark, K.J., Bedell, V.M., Westcot, S.E., Myers, S.R., Moulder, G.L., Thomas, M.J., and Ekker, S.C. (2009). Nicotine response genetics in the zebrafish. Proc Natl Acad Sci U S A 106, 18662-18667.

Plouffe, D., Brinker, A., McNamara, C., Henson, K., Kato, N., Kuhen, K., Nagle, A., Adrian, F., Matzen, J.T., Anderson, P., et al. (2008). In silico activity profiling reveals the mechanism of action of antimalarials discovered in a high-throughput screen. Proc Natl Acad Sci U S A 105, 9059-9064.

Prober, D.A., Rihel, J., Onah, A.A., Sung, R.J., and Schier, A.F. (2006). Hypocretin/orexin overexpression induces an insomnia-like phenotype in zebrafish. J Neurosci 26, 13400-13410.

Prober, D.A., Zimmerman, S., Myers, B.R., McDermott, B.M., Jr., Kim, S.H., Caron, S., Rihel, J., Solnica-Krezel, L., Julius, D., Hudspeth, A.J., et al. (2008). Zebrafish TRPA1 channels are required for chemosensation but not for thermosensation or mechanosensory hair cell function. J Neurosci 28, 10102-10110.

Rawashdeh, O., de Borsetti, N.H., Roman, G., and Cahill, G.M. (2007). Melatonin suppresses nighttime memory formation in zebrafish. Science 318, 1144-1146.

Renier, C., Faraco, J.H., Bourgin, P., Motley, T., Bonaventure, P., Rosa, F., and Mignot, E. (2007). Genomic and functional conservation of sedative-hypnotic targets in the zebrafish. Pharmacogenet Genomics 17, 237-253.

Richetti, S.K., Blank, M., Capiotti, K.M., Piato, A.L., Bogo, M.R., Vianna, M.R., and Bonan, C.D. (2011). Quercetin and rutin prevent scopolamine-induced memory impairment in zebrafish. Behav Brain Res 217, 10-15.

Rihel, J., Prober, D.A., Arvanites, A., Lam, K., Zimmerman, S., Jang, S., Haggarty, S.J., Kokel, D., Rubin, L.L., Peterson, R.T., et al. (2010a). Zebrafish behavioral profiling links drugs to biological targets and rest/wake regulation. Science 327, 348-351.

Rihel, J., Prober, D.A., and Schier, A.F. (2010b). Monitoring sleep and arousal in zebrafish. Methods Cell Biol 100, 281-294. 
This is the pre-peer reviewed version of the following article: Rihel J and Schier AF (2012). Behavioral screening for neuroactive drugs in zebrafish. Developmental Neurobiology, 72(3):373-385., which has been published in final form at http://onlinelibrary.wiley.com/doi/10.1002/dneu.20910/full

Ringholm, A., Fredriksson, R., Poliakova, N., Yan, Y.L., Postlethwait, J.H., Larhammar, D., and Schioth, H.B. (2002). One melanocortin 4 and two melanocortin 5 receptors from zebrafish show remarkable conservation in structure and pharmacology. J Neurochem 82, 6-18.

Rivas-Boyero, A.A., Herrero-Turrion, M.J., Gonzalez-Nunez, V., Sanchez-Simon, F.M., BarretoValer, K., and Rodriguez, R.E. (2011). Pharmacological characterization of a nociceptin receptor from zebrafish (Danio rerio). J Mol Endocrinol.

Ruuskanen, J.O., Peitsaro, N., Kaslin, J.V., Panula, P., and Scheinin, M. (2005). Expression and function of alpha-adrenoceptors in zebrafish: drug effects, mRNA and receptor distributions. J Neurochem 94, 1559-1569.

Sackerman, J., Donegan, J.J., Cunningham, C.S., Nguyen, N.N., Lawless, K., Long, A., Benno, R.H., and Gould, G.G. (2010). Zebrafish Behavior in Novel Environments: Effects of Acute Exposure to Anxiolytic Compounds and Choice of Danio rerio Line. Int J Comp Psychol 23, 4361.

Seibt, K.J., Oliveira Rda, L., Zimmermann, F.F., Capiotti, K.M., Bogo, M.R., Ghisleni, G., and Bonan, C.D. (2010). Antipsychotic drugs prevent the motor hyperactivity induced by psychotomimetic MK-801 in zebrafish (Danio rerio). Behav Brain Res 214, 417-422.

Seifert, R., Wenzel-Seifert, K., Burckstummer, T., Pertz, H.H., Schunack, W., Dove, S., Buschauer, A., and Elz, S. (2003). Multiple differences in agonist and antagonist pharmacology between human and guinea pig histamine H1-receptor. J Pharmacol Exp Ther 305, 1104-1115.

Smith, L.E., Carvan, M.J., 3rd, Dellinger, J.A., Ghorai, J.K., White, D.B., Williams, F.E., and Weber, D.N. (2010). Developmental selenomethionine and methylmercury exposures affect zebrafish learning. Neurotoxicol Teratol 32, 246-255.

Strahle, U., and Grabher, C. (2010). The zebrafish embryo as a model for assessing off-target drug effects. Dis Model Mech 3, 689-692.

Swain, H.A., Sigstad, C., and Scalzo, F.M. (2004). Effects of dizocilpine (MK-801) on circling behavior, swimming activity, and place preference in zebrafish (Danio rerio). Neurotoxicol Teratol 26, 725-729.

Taylor, K.L., Grant, N.J., Temperley, N.D., and Patton, E.E. (2010). Small molecule screening in zebrafish: an in vivo approach to identifying new chemical tools and drug leads. Cell Commun Signal 8, 11.

Thirumalai, V., and Cline, H.T. (2008). Endogenous dopamine suppresses initiation of swimming in prefeeding zebrafish larvae. J Neurophysiol 100, 1635-1648.

Tierney, K.B. (2011). Behavioural assessments of neurotoxic effects and neurodegeneration in zebrafish. Biochim Biophys Acta 1812, 381-389.

Trampus, M., Ferri, N., Adami, M., and Ongini, E. (1993). The dopamine D1 receptor agonists, A68930 and SKF 38393, induce arousal and suppress REM sleep in the rat. Eur J Pharmacol 235, 83-87. 
This is the pre-peer reviewed version of the following article: Rihel J and Schier AF (2012). Behavioral screening for neuroactive drugs in zebrafish. Developmental Neurobiology, 72(3):373-385., which has been published in final form at http://onlinelibrary.wiley.com/doi/10.1002/dneu.20910/full

Webb, K.J., Norton, W.H., Trumbach, D., Meijer, A.H., Ninkovic, J., Topp, S., Heck, D., Marr, C., Wurst, W., Theis, F.J., et al. (2009). Zebrafish reward mutants reveal novel transcripts mediating the behavioral effects of amphetamine. Genome Biol 10, R81.

Wheeler, G.N., and Brandli, A.W. (2009). Simple vertebrate models for chemical genetics and drug discovery screens: lessons from zebrafish and Xenopus. Dev Dyn 238, 1287-1308.

Williams, F.E., and Messer, W.S., Jr. (2004). Muscarinic acetylcholine receptors in the brain of the zebrafish (Danio rerio) measured by radioligand binding techniques. Comp Biochem Physiol C Toxicol Pharmacol 137, 349-353.

Wong, D.T., Perry, K.W., and Bymaster, F.P. (2005). Case history: the discovery of fluoxetine hydrochloride (Prozac). Nat Rev Drug Discov 4, 764-774.

Wong, K., Elegante, M., Bartels, B., Elkhayat, S., Tien, D., Roy, S., Goodspeed, J., Suciu, C., Tan, J., Grimes, C., et al. (2010). Analyzing habituation responses to novelty in zebrafish (Danio rerio). Behav Brain Res 208, 450-457.

Xie, J., Farage, E., Sugimoto, M., and Anand-Apte, B. (2010). A novel transgenic zebrafish model for blood-brain and blood-retinal barrier development. BMC Dev Biol 10, 76.

Yeh, J.R., Munson, K.M., Elagib, K.E., Goldfarb, A.N., Sweetser, D.A., and Peterson, R.T. (2009). Discovering chemical modifiers of oncogene-regulated hematopoietic differentiation. Nat Chem Biol 5, 236-243.

Yokogawa, T., Marin, W., Faraco, J., Pezeron, G., Appelbaum, L., Zhang, J., Rosa, F., Mourrain, P., and Mignot, E. (2007). Characterization of sleep in zebrafish and insomnia in hypocretin receptor mutants. PLoS Biol 5, e277.

Yu, P.B., Hong, C.C., Sachidanandan, C., Babitt, J.L., Deng, D.Y., Hoyng, S.A., Lin, H.Y., Bloch, K.D., and Peterson, R.T. (2008). Dorsomorphin inhibits BMP signals required for embryogenesis and iron metabolism. Nat Chem Biol 4, 33-41.

Zhang, J., Piontek, J., Wolburg, H., Piehl, C., Liss, M., Otten, C., Christ, A., Willnow, T.E., Blasig, I.E., and Abdelilah-Seyfried, S. (2010). Establishment of a neuroepithelial barrier by Claudin5a is essential for zebrafish brain ventricular lumen expansion. Proc Natl Acad Sci U S A $107,1425-1430$.

Zhdanova, I.V. (2006). Sleep in zebrafish. Zebrafish 3, 215-226.

Zhdanova, I.V., Wang, S.Y., Leclair, O.U., and Danilova, N.P. (2001). Melatonin promotes sleep-like state in zebrafish. Brain Res 903, 263-268.

Zon, L.I., and Peterson, R.T. (2005). In vivo drug discovery in the zebrafish. Nat Rev Drug Discov 4, 35-44. 